\title{
Collagen content as a risk factor in breast cancer? A pilot clinical study.
}

\author{
Antonio Pifferi ${ }^{*}{ }^{1,2}$, Giovanna Quarto ${ }^{1}$, Francesca Abbate $^{3}$, Nicola Balestreri $^{4}$, Simona Menna ${ }^{3}$, \\ Enrico Cassano ${ }^{3}$, Rinaldo Cubeddu ${ }^{1,2}$ Paola Taroni ${ }^{1}$ \\ ${ }^{1}$ Dipartimento di Fisica, Politecnico di Milano, Piazza Leonardo da Vinci 32, 20133 Milano, Italy; \\ ${ }^{2}$ Istituto di Fotonica e Nanotecnologie, Consiglio Nazionale delle Ricerche, Piazza Leonardo da \\ Vinci 32, 20133 Milano, Italy; \\ ${ }^{3}$ European Institute of Oncology, Breast Imaging Unit, Via G. Ripamonti, 435, 20141 Milano, Italy; \\ ${ }^{4}$ European Institute of Oncology, Department of Radiology, Milano, Italy
}

\begin{abstract}
A retrospective pilot clinical study on time domain multi-wavelength (635 to $1060 \mathrm{~nm}$ ) optical mammography was exploited to assess collagen as a breast-cancer risk factor on a total of 109 subjects (53 healthy and 56 with malignant lesions). An increased cancer occurrence is observed on the $15 \%$ subset of patients with higher age-matched collagen content. Further, a similar clustering based on the percentage breast density leads to a different set of patients, possibly indicating collagen as a new independent breast cancer risk factor. If confirmed statistically and on larger numbers, these results could have huge impact on personalized diagnostics, health care systems, as well as on basic research.
\end{abstract}

Keywords: Tissue diagnostics, time-resolved diffuse optical imaging, breast cancer, collagen, risk assessment.

\section{INTRODUCTION}

The identification of risk factors for breast cancer is a relevant open quest. The chance to identify clusters of subjects at high risk permits to foresee personalized diagnostics paths on smaller groups - not sustainable in population-wide screening programs. Indeed, the early diagnosis of breast cancer (size $<1 \mathrm{~cm}$, no lymphnode involvement) leads to an impressive $95 \%$ complete remission rate. Breast density is recognized as a strong and independent risk factor for breast cancer and is usually quantified by X-ray mammography. Recently, optical techniques have been shown to offer a valid non-invasive and possibly cost-effective alternative for the assessment of breast density even at young age $\mathrm{e}^{1-3}$.

In this paper we explore an even more ambitious goal, which is to investigate whether optical techniques can be used directly to assess breast cancer risk factors and not just indirectly by correlation with breast density. More specifically, we address collagen - quantified by time-resolved diffuse optical spectroscopy ${ }^{4}$ - as a potential independent risk factor for breast cancer ${ }^{5}$.

\section{MATERIALS AND METHODS}

\subsection{Instrument set-up}

Time-resolved transmittance scans are performed at 7 wavelengths $(635,685,785,905,930,975,1060 \mathrm{~nm})$ using pulsed diode lasers and time-correlated single photon counting ${ }^{4}$. The compressed breast is scanned continuously recording data every $\mathrm{mm}$. The central region of the breast, far from boundaries, is used for assessment of breast optical properties. A schematics of the instrument is shown in Fig. 1.

\subsection{Patient study}

For the purpose of this investigation, a total of 109 patients were included following criteria of i) availability of digital mammography for percentage breast density assessment; ii) absence of technical failure (e.g. poor signal); iii) exclusion

\footnotetext{
* Further author information:

A. Pifferi e-mail: antonio.pifferi@fisi.polimi.it — P. Taroni e-mail: paola.taroni@polimi.it
} 
of patients with benign lesions or not clearly classified malignant lesions (e.g. DCS). Two subsets of 53 healthy subjects (normal) and 56 patients bearing a malignant lesion (cancer) were extracted.

\subsection{Data analysis}

Tissue composition and structure is obtained directly from time-resolved transmittance curves using a spectrally constrained global fitting procedure on the key absorber concentrations (oxy- and deoxy-hemoglobin, water, lipids, and collagen) together with the scattering amplitude $a$ and power $b$. The collagen absorption spectrum was obtained using a broadband time-resolved diffuse optical spectrometer ${ }^{6}$ on bovine tendon collagen I powder purchased from Sigma ${ }^{7}$.

An experienced radiologist evaluated percentage mammographic density using a fully automated procedure ${ }^{8}$.

Age-matched distributions of collagen and density are obtained by dividing both the collagen content and the percentage density by the corresponding theoretical age-matched value obtained by a linear interpolation on the normal distribution.

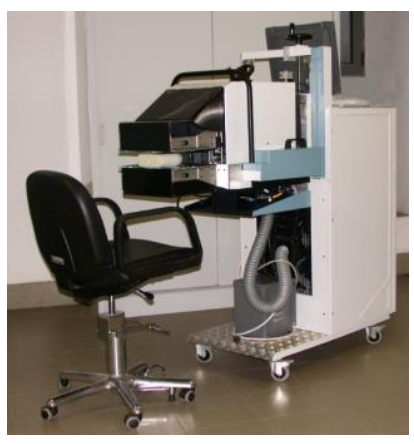

- Continuous movement: stepping motors \& Counter/Timer PC board

- Maximum scan area: $180 \mathrm{~mm}$ x $240 \mathrm{~mm}$

- Step interval: $1 \mathrm{~mm}$

- Measurement time: 25 ms per point

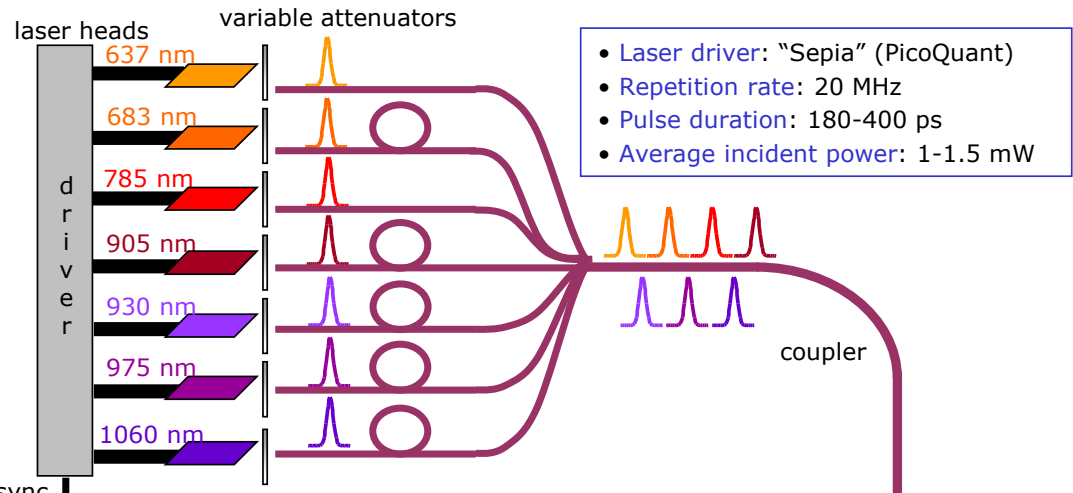

sync $\quad$ Photomultipliers (Hamamatsu, KK): VIS: R5900U-01-L16, 150 ps TTS, $\lambda<850 \mathrm{~nm}$ NIR: H7422P-60, 450 ps TTS, $\lambda<1100 \mathrm{~nm}$

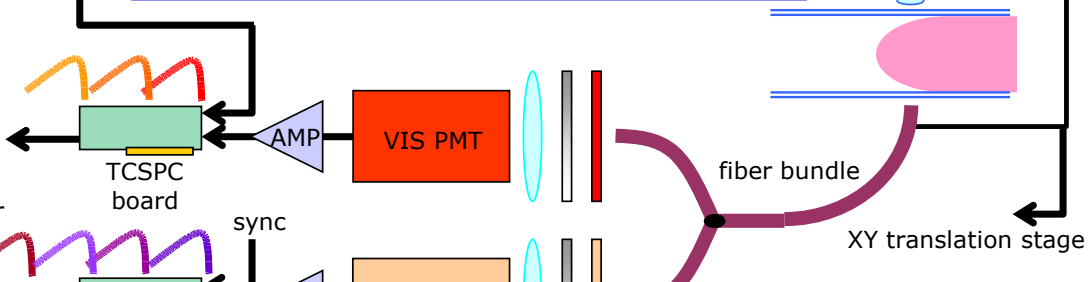

- TCSPC board (SPC-134, Becker \& Hickl): 4 independent channels $16 \mathrm{MHz}$ max count rate

Figure 1. Schematics of the time-domain optical mammograph used in the clinical study.

\section{RESULTS AND DISCUSSION}

In principle, the investigation of a new potential breast cancer risk factor would require a long longitudinal study involving a high number of subjects to understand whether factor $\mathrm{X}$ detected at age $\mathrm{Y}$ is correlated with a later occurrence of cancer. Clearly, such a huge trial can be undertaken only when the initial hypothesis is explored on a much smaller scale. The approach we pursue here for a first insight is to address the question retrospectively, i.e. to find out whether subjects bearing a breast lesion have some signs in their healthy breast suggesting a higher risk factor. For this purpose, we exploited the outcome of a clinical study on optical mammography initially designed for the aim of differentiating optically malignant from benign lesions ${ }^{9}$. Just two groups are identified here, i.e. normal (healthy subject) and cancer (bearing a malignant lesion).

Figure 2 displays the box plot of the collagen content and of the percentage mammographic density for the two groups. There is a slightly higher mean collagen content in the cancer group as compared to the normal one, yet these differences 
are meaningless considering the high dispersion of the data. At a first sight one could conclude that the chance to use collagen as a breast cancer risk factor is deemed to fail. Nevertheless, a similar pattern is observed also for the percentage density, for which correlation with higher cancer risk is clearly demonstrated in literature. The clue here is that the number of subjects that get cancer because of their higher breast density is just a fraction of the total population. Thus its impact on the mean values is quite diluted.

In order to investigate the possible role of collagen as a risk factor for a specific sub-group of the whole population, we mimicked - at first order - the known results reported for breast density. First, age-matched effective collagen values were derived dividing the measured quantity by the theoretical average age-matched estimates as described in Section 2.3. Then, the cohort of subjects with a higher collagen content are compared to the distribution observed on the whole population.

Figure 3 shows the plot of the occurrence of cancer (red solid line) and normal (blue dotted line) lesions in the cohort of subjects with the highest age-matched collagen content. The threshold indicates the percentage of the total population that are included in the cohort. For a threshold around 15\%, the high-collagen cohort exhibit a largest incidence of cancer lesions (about $80 \%$ ) as compared to the total population included in this study that have a cancer incidence of $50 \%$. This increase is large since we have to consider that the maximum incidence is necessarily saturated at $100 \%$.

To translate these numbers into increased risk factor for a standard population, where the average occurrence of cancer lesions is obviously much lower, a proper non-linear model must be applied. Also, more refined analysis is needed to assess the statistical significance of these results.
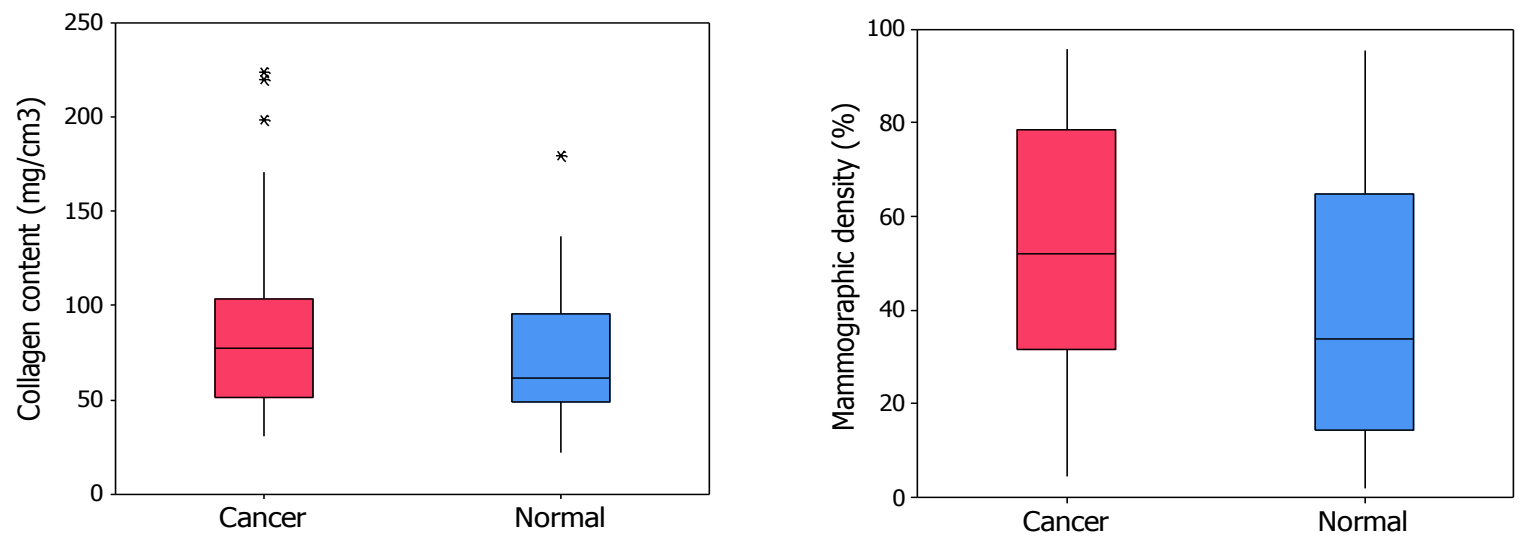

Figure 2. Box plot of the collagen content (left) and percentage breast density (right) for cancer and normal subjects.

\section{CONCLUSION}

In conclusion, in a retrospective pilot clinical study involving 109 patients we show that, sorting subjects on the basis of age-matched collagen content, it is possible to identify an increased cancer occurrence as compared to the reference value on the whole population considered in the study. This means that collagen could be considered for screening purposes to identity women at higher risk for breast cancer. This study is still preliminary and will be further investigated on statistical basis and compared with results obtained using breast density as classification index. These results are consistent with what inferred from recent studies showing possible involvement of collagen in breast cancer onset and progression $^{4}$, and will open new screening potential if confirmed on a larger scale. Impressive growth on photonics components are now opening the way to deployment of small, cost-effective, and portable time-resolved diffuse optics devices $^{10,11}$, which could be used in simple and operator independent breast cancer risk screening programs at the level of the general practitioner. 


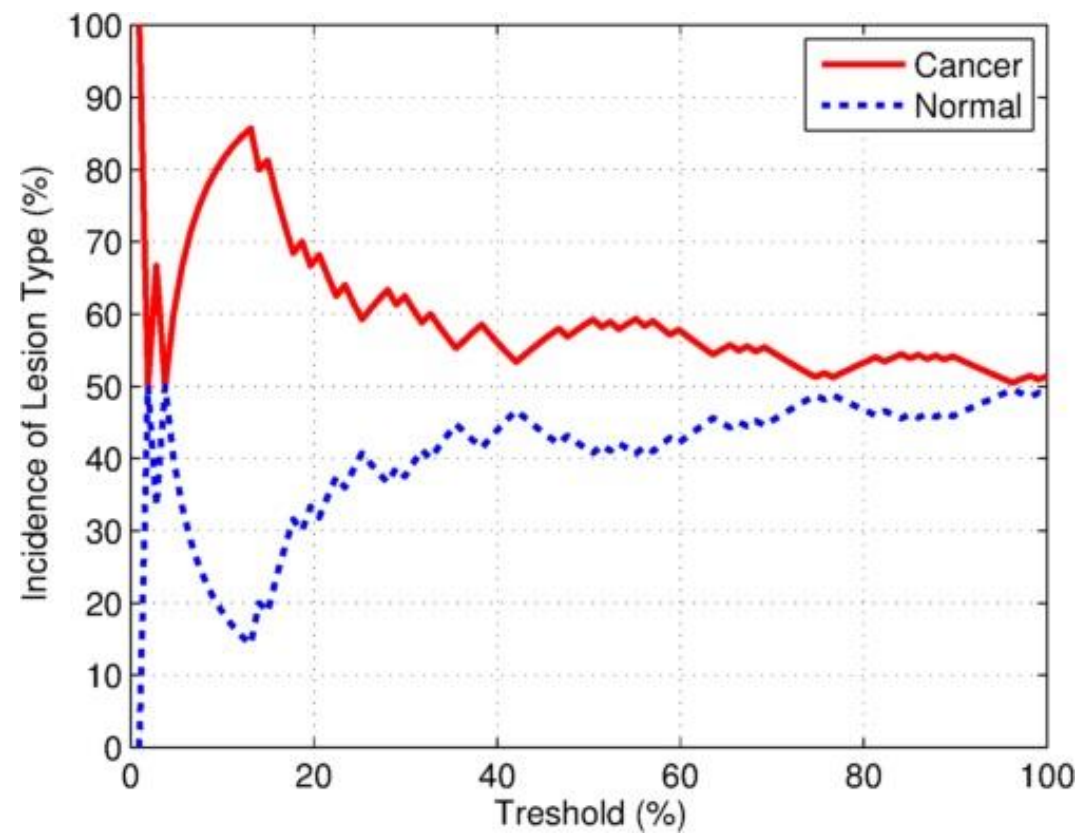

Figure 3. Age normalized collagen $v s$. breast density for the cancer and the normal subjects.

\section{REFERENCES}

[1] Taroni, P., Pifferi, A., Quarto, G., Spinelli, L., Torricelli, A., Abbate, F., Balestreri, N., Menna, S., Cassano, E. and Cubeddu, R., "Non-invasive assessment of breast cancer risk using time-resolved diffuse optical spectroscopy," J. Biomed. Opt.15, 060501 (2010).

[2] Blackmore, K. M., Knight, J. A. and Lilge, L., "Association between transillumination breast spectroscopy and quantitative mammographic features of the breast," Cancer Epidemiol. Biomarkers Prev. 17, 1043-1050 (2008).

[3] Taroni, P., Quarto, G., Pifferi, A., Ieva, F., Paganoni, A. M., Abbate, F., Balestreri, N., Menna, S., Cassano, E., et al., "Optical identification of subjects at high risk for developing breast cancer," J. Biomed. Opt. 18(6), 060507, (2013).

[4] Taroni, P., Pifferi, A., Salvagnini, E., Spinelli, L., Torricelli, A. and Cubeddu, R., "Seven-wavelength timeresolved optical mammography extending beyond $1000 \mathrm{~nm}$ for breast collagen quantification," Opt. Expr. 17, 15932-15946 (2009).

[5] Couzin, J., "Dissecting a hidden breast cancer risk," Science 309, 1664-1666 (2005).

[6] Pifferi, A., Torricelli, A., Taroni, P., Comelli, D., Bassi, A.., Cubeddu, R., "Fully automated time domain spectrometer for the absorption and scattering characterization of diffusive media," Rev. Sci. Instrum. 78(5), 053103, (2007).

[7] Taroni, P., Bassi, A., Comelli, D., Farina, A., Cubeddu, R.., Pifferi, A., "Diffuse optical spectroscopy of breast tissue extended to $1100 \mathrm{~nm}$, , J. Biomed. Opt. 14(5), 054030 (2009).

[8] Tagliafico, A., Tagliafico, G., Tosto, S., Chiesa, F., Martinoli, C., Derchi, L.E. and Calabrese, M., "Mammographic density estimation: Comparison among BI-RADS categories, a semi-automated software and a fully automated one," Breast 18, 35-40 (2009).

[9] Quarto, G., Spinelli, L., Pifferi, A., Torricelli, A., Cubeddu, R., Abbate, F., Balestreri, N., Menna, S., Cassano, E., et al., "Estimate of tissue composition in malignant and benign breast lesions by time-domain optical mammography," Biomed. Opt. Express 5(10), 3684-3698, (2014).

[10] A. Dalla Mora, D. Contini, S. Arridge, F. Martelli, A. Tosi, G. Boso, A. Farina, T. Durduran, E. Martinenghi, A. Torricelli, and A. Pifferi, "Towards next-generation time-domain diffuse optics for extreme depth penetration and sensitivity," Biomed. Opt. Express, 6(5), 1749 (2015).

[11] A. Dalla Mora, E. Martinenghi, D. Contini, A. Tosi, G. Boso, T. Durduran, S. Arridge, F. Martelli, A. Farina, A. Torricelli, and A. Pifferi, "Fast silicon photomultiplier improves signal harvesting and reduces complexity in time-domain diffuse optics," Opt. Express, 23(11), 13937 (2015). 